\title{
Clase, género y emancipación en Erik Wright y Pierre Bourdieu
}

Gonzalo Seid ${ }^{1}$

\section{Universidad de Buenos Aires}

\section{Ensayo}

Material original autorizado para su primera publicación en Journal de Ciencias Sociales, Revista Académica de la Facultad de Ciencias Sociales de la Universidad de Palermo.

\section{Recibido: 27-09-2017}

Aceptado: 07-02-2018

Resumen: En este trabajo se desarrollarán algunos elementos conceptuales sobre la articulación entre clase y género como factores de desigualdad y opresión. Se presentarán los enfoques teóricos de Erik Olin Wright y Pierre Bourdieu sobre las clases sociales, se examinarán sus aportes sobre la inclusión del género en el análisis de clase y se mencionarán algunas de sus reflexiones en torno a las perspectivas de emancipación. Luego de presentar los principales aportes de cada autor sobre clase, género y emancipación, se propone una breve confrontación de ambas perspectivas teóricas respecto a algunos de los supuestos básicos y al modo de abordar las problemáticas de investigación.

Palabras clave: clase; género; emancipación; desigualdades.

\section{Social class, gender and emancipation in Erik Wright and Pierre Bourdieu}

Abstract: The conceptual notions that articulate class and gender, considered as factors of oppression and inequality, are expounded in this paper. For this purpose, Erik Olin Wright's and Pierre Bourdieu's theoretical approaches on social classes are presented, focusing on their contributions in the incorporation of gender in the analysis of social class, and their considerations about emancipation prospects. Each author's main contributions on class, gender and emancipation are explained in detail and later their theoretical perspectives are briefly contrasted, considering some of their basic assumptions and the way they address the issues being studied.

Keywords: class; gender; emancipation; inequalities.

\footnotetext{
1 Sociólogo. Magíster en Investigación en Ciencias Sociales (UBA). Correo electrónico: gonzaloseid@gmail.com
} 


\section{Introducción}

En la sociología de la estratificación social la clase social ha sido tradicionalmente operacionalizada a partir de la ocupación del jefe de hogar, cabeza de familia o principal sostén económico, que durante el siglo XX, cuando se desarrolló este campo de investigación, ha sido por lo general un varón. El punto de vista convencional ha sido sostenido por importantes referentes en la temática como John Goldthorpe (1983), quien ha argumentado que las unidades de análisis para los estudios de estratificación y movilidad social deben ser los hogares, que los miembros de los hogares comparten su posición en la estructura social y oportunidades de vida, y que dada la situación de desventaja y dependencia de las mujeres, lo más conveniente es medir la clase del hogar a partir de indicadores referidos a las ocupaciones de los hombres, excepto cuando el hogar carezca de jefe varón. Este enfoque ha sido criticado por hacer invisible la clase de las mujeres, la heterogeneidad de clase que puede existir en el interior del hogar y el papel del género atravesando las relaciones de clase (Sorensen, 1994; Salido Cortés, 2001; Gómez Rojas, 2010). Asimismo, con la extensión de nuevas configuraciones familiares desde fines del siglo $X X$, el enfoque convencional resulta cada vez más insatisfactorio.

Ahora bien, la incorporación de las mujeres y los distintos miembros de las familias en el análisis de clase plantea algunos desafíos teóricos y metodológicos, precisamente aquellos que no eran problematizados y se daban por sentados en la perspectiva tradicional. Si generalmente se acepta que la unidad de análisis más adecuada para estudiar la clase social es la familia o el hogar, ¿es posible que existan diferentes posiciones de clase entre los miembros de una familia? ¿Cómo puede incluirse la dimensión de género en el análisis de clase? ¿Qué enfoques teóricos permiten hacerlo de manera consistente con sus conceptualizaciones de la clase? A partir de estos interrogantes como disparadores de la reflexión teórica y metodológica, en este artículo se revisan las aproximaciones de Erik Olin Wright y Pierre Bourdieu al análisis de clase. Ambos exponentes de la sociología contemporánea han realizado aportes teóricos e investigaciones empíricas sobre las clases sociales que pueden tomarse como alternativas a la perspectiva convencional que continúa prevaleciendo en muchos estudios de estratificación y movilidad social. Wright y Bourdieu no se han concentrado especialmente en incorporar la dimensión de género en el análisis de clase, pero en sus obras pueden encontrarse modos de entender las clases que abren posibilidades de complejizar el análisis de clase en esa dirección.

Sobre la teoría de las clases de Bourdieu ha habido numerosas síntesis y sistematizaciones posteriores a la obra del autor (Álvarez Souza, 1996; Castón Boyer, 1996; Weininger, 2005; Wacquant, 2013). En el caso de Erik Wright las sistematizaciones están principalmente en la propia obra del autor o en producciones colectivas de las que participó 
(Carabaña, 1995; Wright, 2005), aunque se encuentran habitualmente referencias a sus aportes como principal exponente del "neomarxismo" para la investigación empírica cuantitativa en estratificación social (Rivas Rivas, 2008; Riveiro y Castañeira, 2009). En la investigación de la temática de estratificación y movilidad social, además de la teoría clásica funcionalista (cuya gravitación se ha prolongado en este campo más que en otras temáticas), el enfoque neoweberiano de Goldthorpe (1992) es probablemente el más extendido y, como alternativas, el enfoque neodurkheimiano de Grusky (2007), el neomarxista de Wright y el de Bourdieu. Al tratarse de enfoques teóricos distintos, a menudo se considera, atinadamente, que en las investigaciones empíricas debería optarse por alguno de estos marcos conceptuales. En este campo tradicionalmente ha existido una propensión a la estandarización en los tipos de preguntas, los instrumentos de relevamiento y las formas de análisis, que ha llevado a omitir la cuestión de género o plantearla de maneras no del todo satisfactorias. Por ello, el análisis y la puesta en diálogo de dos de las alternativas de mayor relevancia teórica puede ser útil para ponderar sus alcances y límites para la investigación sobre clase y género.

A continuación, se desarrollarán algunos de los principales aportes de Wright y Bourdieu sobre la articulación entre clase y género como ejes de desigualdad, poniendo el acento en reconstruir las principales definiciones para la clarificación conceptual de sus enfoques teóricos. Asimismo, para hacer explícitos los supuestos teórico-políticos de sus modos de entender las desigualdades sociales se hará referencia a las concepciones sobre la emancipación social que sus perspectivas teóricas contienen, entendiendo que el horizonte de transformaciones sociales deseables para eliminar opresiones forma parte de las teorías, a la vez que éstas contribuyen a imaginarlo.

En el primer apartado se desarrollarán las conceptualizaciones sobre clases sociales desde el enfoque neomarxista de Wright; la articulación entre clase y género y, para finalizar, su mirada sobre la emancipación. En el segundo apartado, se seguirá la misma secuencia expositiva para presentar la perspectiva de Bourdieu. Por último, se pondrán en diálogo y discusión los abordajes de ambos autores.

\section{La teoría de clases de Erik Olin Wright}

En el siglo XX se desarrollaron variadas corrientes de pensamiento herederas del marxismo -entre las más difundidas, la teoría crítica de la Escuela de Frankfurt y las contribuciones teórico-políticas de Antonio Gramsci- que criticaron y retomaron distintos aspectos. Las críticas al mecanicismo, al materialismo vulgar y al economicismo orientaron la teorización hacia las esferas de la cultura y la ideología, que cobraron una renovada centralidad. Frente a la impronta filosófica e ideológica prevaleciente en los marxismos, en la 
década de 1980 surgió el marxismo analítico, corriente de raigambre anglosajona que se propuso reconstruir el marxismo manteniendo un fuerte compromiso con la rigurosidad científica de estándares convencionales. El "grupo de Septiembre" de marxismo analítico, del que Erik Wright formó parte, se reunía a discutir la teoría marxista con preocupaciones en torno a la consistencia lógica de las definiciones y su adecuación al conocimiento científico acumulado, procurando desechar las categorías metafísicas incomprobables y los resabios dialécticos hegelianos. Entre los puntos en común de estos autores se destacan la orientación analítica, la vinculación del marxismo con la teoría de la elección racional y el interés en superar la mera especulación teórica, lo que se conseguía mediante una investigación empírica sistemática que confronte la teoría con la realidad y sometimiento a crítica y revisión constante.

Wright ha procurado elaborar un concepto de clase que, desde la tradición marxista, permita describir las variaciones de la estructura de clases en las sociedades capitalistas. Un primer punto que cabe señalar es la crítica a los enfoques de clase gradacionales o de sentido común, tales como las jerarquías de ingreso o prestigio, característicos de los estudios de estratificación social centrados en los atributos individuales y el logro de status, de raigambre funcionalista. Tanto los neoweberianos, fundamentalmente Goldthorpe, como los neomarxistas entre los que se encuentra Wright, coinciden en que la distribución de recompensas es un resultado de los procesos de clase y que no explica las relaciones sociales subyacentes, los conflictos y los mecanismos específicos que generan desigualdades. El carácter relacional de las clases sociales hace que sólo puedan ser definidas en términos de relaciones sociales con otras clases y no a partir de agregados ocupacionales que no constituyen agrupaciones de importancia sociológica real.

Dentro de las concepciones relacionales, Wright (2005) diferencia la tradición marxista de la weberiana partiendo de la habitual distinción entre las definiciones de clase basadas en las relaciones de producción y las basadas en las relaciones de mercado. Wright señala el carácter "doblemente relacional" de la definición marxista, que no se restringe a lo relacional del intercambio de bienes y la monopolización de oportunidades, sino que incluye las interacciones entre trabajadores y capitalistas en la producción, ámbito donde ocurre primariamente la explotación a partir de la apropiación capitalista del excedente de trabajo. En las investigaciones empíricas sobre estructura de clases ha tendido a existir cierta confluencia entre neomarxistas y neoweberianos (Longhi, 2005) puesto que ambos toman la ocupación como principal indicador de clase y el neomarxismo incorporó otras dimensiones además de la relación con los medios de producción, como las credenciales educativas y el control sobre el proceso productivo. Tal vez la principal diferencia sea que para los marxistas las relaciones entre las clases son relaciones de producción, en las que necesariamente tiene 
lugar la explotación, mientras que para los weberianos esta sería sólo una posibilidad (Wright, 2002).

Las relaciones sociales de producción y el concepto de explotación continúan siendo aspectos decisivos de la tradición marxista que Wright conserva. Este autor ha intentado renovar la definición tradicional de clase basada en la propiedad de los medios de producción, combinando los conceptos de explotación y dominación. La dominación refiere a la capacidad de controlar las actividades de otros, mientras que la explotación supone la apropiación de beneficios económicos a partir del trabajo de los dominados (Wright, 1995). Cuando existe explotación, el grupo explotador no sólo se beneficia por la privación o restricción del acceso de otros a ciertos recursos o posiciones, sino que además controla el trabajo de aquellos en beneficio propio puesto que necesita del esfuerzo del explotado. A diferencia de la perspectiva weberiana, para el marxismo no sólo se observan desigualdades en el bienestar material y clausura social, sino un mecanismo causal que traduce la exclusión del acceso a recursos en diferencias de bienestar, mediante la apropiación de los frutos del trabajo de los explotados por parte de quienes controlan los recursos productivos. La explotación puede entenderse como un conflicto de suma cero en que todo aumento en la ganancia implica una mayor tasa de explotación, por lo cual explotadores y explotados tienen necesariamente intereses objetivos antagónicos.

En la primera versión de su esquema de clases, Wright (1983) ha identificado tres dimensiones de las relaciones sociales de producción: el control sobre el capital monetario, el control sobre los medios físicos de producción y el control sobre la fuerza de trabajo a partir de la supervisión y disciplina en el proceso laboral. Las posiciones fundamentales en las relaciones de clase son la burguesía, que posee los medios de producción y controla las tres dimensiones de las relaciones de producción; el proletariado, que carece de propiedad y de todo control, y la pequeña burguesía que posee y controla medios de producción, pero no la fuerza de trabajo de otros.

En medio de las clases principales existen posiciones que son conceptualizadas como situaciones contradictorias de clase, noción con la que Wright pretende avanzar hacia la transformación de la categoría ideológica de clase media en un concepto científico capaz de dar cuenta de la ausencia de polarización de clases prevista por Marx (Marx y Engels, 1998 [1848]; Marx, 2003 [1852]). La idea desarrollada por Wright consiste en que "no todas las posiciones de la estructura social pueden considerarse como firmemente enraizadas en una única clase: ciertas posiciones ocupan objetivamente situaciones contradictorias entre las clases" (1983, p. 23). Entre la burguesía y el proletariado, los directivos y supervisores controlan los medios de producción y la fuerza de trabajo, aunque no son propietarios del capital. Entre el proletariado y la pequeña burguesía, los empleados semiautónomos de 
posiciones técnicas profesionales preservan un control real sobre su proceso de trabajo -sobre lo que producen y cómo lo producen-aunque no poseen ni controlan el capital y deben vender su fuerza de trabajo. Entre la burguesía y la pequeña burguesía, se ubican los pequeños empleadores que, aunque son propietarios de los medios de producción y contratan fuerza de trabajo, siguen siendo productores directos autoempleados.

En una segunda versión de su mapa de clases, Wright (1994[1985]) procura poner mayor énfasis en los mecanismos de explotación para la definición de las clases. A partir de los desarrollos teóricos de John Roemer (1989), Wright distingue cuatro tipos de bienes cuya propiedad y control implican diferentes tipos de explotación, lo que da lugar a la posibilidad de que algunas clases sean explotadas mediante un mecanismo a la par que son explotadoras mediante otro. Los bienes cuya propiedad y control están en la base de la explotación son la fuerza de trabajo (explotación feudal), el capital (explotación capitalista), los bienes de organización —vinculados a la coordinación e integración de la división del trabajo- (explotación estatal) y los bienes de cualificación —vinculados a las credenciales educativas y la calificación de las tareas- (explotación socialista). Como resultado de la combinación de las distintas situaciones respecto a los bienes de producción, de organización y de cualificación, Wright elabora una tipología de doce clases. Entre los propietarios de medios de producción, se ubica la burguesía que posee suficiente capital para contratar trabajadores y no trabajar, los pequeños empleadores que poseen suficiente capital para contratar trabajadores pero deben trabajar, y la pequeña burguesía que posee suficiente capital para trabajar autónomamente pero no para contratar trabajadores. Entre los no propietarios de medios de producción surgen nueve posiciones de clase de la combinación de las posiciones asalariadas respecto a los bienes organizacionales (directivos, supervisores y no gerenciales) y a los bienes de cualificación (expertos, empleados calificados y empleados no calificados).

Tabla 1. Tipología de doce posiciones de clase

\begin{tabular}{|c|c|c|c|c|}
\hline Propietarios & \multicolumn{3}{|l|}{ Asalariados } & \\
\hline Burguesía & $\begin{array}{l}\text { Directivos } \\
\text { expertos }\end{array}$ & $\begin{array}{l}\text { Directivos } \\
\text { calificados }\end{array}$ & $\begin{array}{ll}\text { Directivos } & \text { no } \\
\text { calificados } & \end{array}$ & \multirow{3}{*}{$\begin{array}{l}\mathbf{+} \\
\text { Bienes de } \\
\text { organización }\end{array}$} \\
\hline $\begin{array}{l}\text { Pequeños } \\
\text { empleadores }\end{array}$ & $\begin{array}{l}\text { Supervisores } \\
\text { expertos }\end{array}$ & $\begin{array}{l}\text { Supervisores } \\
\text { calificados }\end{array}$ & $\begin{array}{l}\text { Supervisores } \\
\text { no calificados }\end{array}$ & \\
\hline \multirow[t]{2}{*}{$\begin{array}{l}\text { Pequeña } \\
\text { burguesía }\end{array}$} & $\begin{array}{l}\text { Expertos no } \\
\text { directivos }\end{array}$ & $\begin{array}{l}\text { Obreros } \\
\text { calificados }\end{array}$ & Proletarios & \\
\hline & \multicolumn{3}{|c|}{ Bienes de cualificación - } & \\
\hline
\end{tabular}

Fuente: Wright (1994).

Para Wright resulta fundamental que la clarificación de la estructura de clases sea puesta en relación con la formación y la lucha de clases. A continuación, se sintetizan los principales vínculos entre una serie de conceptos que aluden a aspectos de las clases y que, 
articulados, constituyen hipótesis sobre las variadas modalidades de determinación que ocurren en estos procesos sociales entre y dentro de las clases. La estructura de clases, en tanto estructura de relaciones sociales entre las clases, constituye el mecanismo básico que determina el acceso a los recursos y las capacidades para actuar de una formación de clase. La lucha de clases se ve limitada por la estructura de clases con respecto a sus actores y objetivos potenciales, pero también la lucha puede transformar la estructura de clases. Los intereses de clase son los objetivos reales de lucha de las clases bajo el supuesto de que no existan mistificaciones y distorsiones ideológicas. La conciencia de clase consiste en la comprensión adecuada de los intereses de clase, que en el caso de los trabajadores implica la toma de conciencia de que es necesario transformar la estructura de clases para cambiar su poder. Los intereses inmediatos, referidos a las condiciones de existencia dentro del capitalismo, pueden diferir al interior de la clase obrera, por ejemplo, entre trabajadores calificados y no calificados, o entre trabajadores y trabajadoras. Los intereses fundamentales son aquellos que se definen entre modos de producción y que aglutinan al conjunto de la clase trabajadora en torno al socialismo en tanto interés objetivo.

Por último, la formación de clase deviene de la estructura real de las relaciones sociales dentro de las clases. Las capacidades de clase se definen por las relaciones sociales dentro de una clase que unifican a los agentes, estas capacidades conforman las consecuencias de una formación de clase para la lucha de clases. La lucha de clases, a su vez, puede transformar las capacidades organizativas y estructurales de una formación de clase.

\section{Clase y género}

Aunque en las sociedades actuales existan efectos recíprocos y sistemáticos entre las relaciones de género y las relaciones de clase, Wright propone mantener la distinción entre ambas dimensiones. Si bien las relaciones de género no están determinadas únicamente por las relaciones de clase, la estructura de clases establece límites de variaciones posibles. Las relaciones de género a su vez pueden influir en la distribución de los individuos en las clases y producir divisiones dentro de las clases. El autor no considera satisfactorio sostener que las relaciones de género puedan considerarse como un tipo específico de relación de clase.

Un aporte especialmente relevante de Wright para la articulación de clase y género es el concepto de relaciones mediatas de clase, que proporciona una forma concreta de abordar en el análisis de clase el problema de la interrelación entre clase, estructura familiar y género. Wright (1983) considera que para establecer la posición de aquellas categorías de personas que no son activos remunerados, el criterio reside en su relación con los intereses 
materiales fundamentales de clase. De este modo, se amplía la definición de pertenencia a una clase para incluir a aquellas personas que, si bien no ocupan una posición en las relaciones sociales de producción, están ligados a una clase por su familia o por trayectorias de clase. Por ejemplo, las amas de casa esposas de obreros deben ser clasificadas en la clase obrera porque ellas también tienen el interés de clase fundamental en el socialismosin que esto implique negar que estén oprimidas en la división sexual del trabajo y puedan tener otros intereses adicionales como mujeres-. Aun si se considerara que las amas de casa son trabajadoras domésticas que adquieren su posición de clase obrera sobre la base de las relaciones de género, ha de reconocerse que las diferentes clases de sus maridos no pueden resultar indiferentes respecto a sus propias posiciones.

En definitiva, las posiciones e intereses de clase pueden estar condicionados no sólo por los diversos tipos de relaciones de producción sino también por las redes de relaciones sociales. Las redes de parentesco y las estructuras familiares constituyen el ejemplo más representativo, pero también la pertenencia a ciertas comunidades y la relación con el Estado pueden constituir bases de relaciones mediatas de clase que vinculan los intereses materiales con el proceso de explotación. Los niños, las amas de casa que no trabajan remuneradamente, los desempleados, los jubilados y los estudiantes pertenecen a una clase social a partir de sus relaciones mediatas con el sistema productivo.

Desarrollando más esta idea, Wright (1992) sostiene que para elaborar un concepto de estructura de clase de nivel micro, capaz de aprehender cómo se organizan en relaciones de clase las vidas individuales, el vínculo simple entre individuos en empleos y clases tiene que ser modificado de varias maneras. Una de ellas consiste en incluir en la descripción de la estructura de clases no sólo las relaciones directas de clase que se corresponden con los empleos, sino también las posiciones mediatas. En vez de preguntarse cuál es el posicionamiento de clase de una persona, debería preguntarse cuál es su ubicación dentro de una red de relaciones de clase directas y mediadas, estudiando así "individuos en familias", lo que constituye una mejor aproximación a la estructura de clase en el capitalismo contemporáneo. Ya no se trata de resolver el problema de la clasificación de los que no son activos remunerados, sino que es posible complejizar el análisis de clase para los hogares en que ambos esposos son activos, con la posibilidad de que maridos y mujeres tengan posiciones directas de clase diferentes y por ende también distintas posiciones mediatas.

Un tipo de situación relevante que puede ocurrir consiste en que una trayectoria de clase esté vinculada a la puesta en marcha de mecanismos que estaban presentes en potencia en una relación mediata de clase, por ejemplo mediante una herencia por fallecimiento. Con respecto al caso de las personas casadas, para pensar la temporalidad de las posiciones de clase, Wright propone el concepto de "clase sombra", es decir, la clase que 
tendrían en caso de que el individuo se separe o enviude, lo cual resulta especialmente útil dado el posible y frecuente desfasaje entre la posición de clase individual de varones y mujeres, por ende, de la posición directa y la posición mediata de cada uno de ellos.

Puesto que no resulta satisfactorio identificar la posición de clase de las esposas con la de sus maridos, ni tampoco hacer de la clase un atributo individual, la estructura de clases debería comprenderse como el conjunto de relaciones directas y mediatas. De este modo, maridos y esposas pueden compartir la misma posición de clase en tanto unidad familiar, por ejemplo en lo que respecta al consumo y estrategias familiares, mientras que en otros aspectos pueden mantener posiciones de clase distintas; por lo cual los intereses materiales de los individuos constituyen un producto de esta combinación.

\section{Emancipación}

Wright (2010) entiende por emancipación la eliminación de cierta opresión o situación en que se priva a un grupo de la realización de valores relevantes, lo que produce diferencias sistemáticas entre categorías de actores sociales respecto a su poder social y bienestar material. Para el autor, tanto el marxismo como el feminismo son teorías emancipatorias de la opresión de clase y de género respectivamente.

Mientras que en el marxismo se ha debatido acerca de si es realmente posible una sociedad sin clases, en el feminismo ha habido, según Wright (1994), cierto silencio sobre la viabilidad de eliminar la dominación masculina. Este silencio se vincula a que se da por sentada la viabilidad de la igualdad de género, a diferencia de cierto escepticismo entre los marxistas acerca de la factibilidad de las formas más igualitarias del proyecto emancipatorio de clases. El autor sugiere que las transformaciones en las relaciones de clase en el capitalismo avanzado podrían explicar por qué la eliminación de ciertas formas institucionalizadas de dominación masculina parece haber llegado a ser históricamente posible.

Algunas formas prefigurativas de la igualdad de género pueden experimentarse parcialmente en micro-contextos dentro de la sociedad con dominación masculina, experiencias que parece plausible extrapolar a la sociedad como un todo, puesto que no existen contradicciones de género como tales en la coordinación de nivel macro. En cambio, las relaciones solidarias entre trabajadores no pueden extrapolarse directamente a una imagen de sociedad sin opresión de clase, puesto que la producción socialista requiere una coordinación activa y estructuras institucionales que generen dinámicas de macro-nivel.

En la tradición marxista ha habido desarrollos teóricos que han asumido formas relativamente deterministas y que han postulado leyes del movimiento social que tenderían a 
dirigir el cambio hacia trayectorias específicas, por ejemplo la célebre ley sobre la tendencia decreciente de la tasa de ganancia (Marx, 2006 [1867]), que obstaculizaría la reproducción del capitalismo y llevaría a su colapso. Cuando han perdido vigencia las teorías sobre la tendencia inherente al capitalismo de dirigirse hacia un colapso catastrófico, Wright considera que el marxismo necesita una teoría positiva y más sistemática de la sociedad sin clases para que el proyecto emancipatorio recupere credibilidad. En las tradiciones teóricas feministas, en cambio, el determinismo no ha sido una preocupación central para que la emancipación tenga credibilidad, puesto que la destrucción del patriarcado no derivaría de sus contradicciones internas, sino que dependería más de la agencia, la conciencia y las luchas.

\section{La teoría de clases de Pierre Bourdieu}

Pierre Bourdieu (2012 [1979]) sostiene que las clases sociales son construcciones analíticas, pero bien fundadas en la realidad. Desde su perspectiva, lo que existe no son "clases sociales" tal como se las concibe en el modo de pensar sustancialista, sino un espacio social multidimensional, con diversos factores de diferenciación, poderes sociales o formas de capital: económico —en su acepción habitual—, cultural —conocimientos incorporados, credenciales educativas y bienes culturales—, social —en el sentido de recursos basados en vínculos y pertenencia grupal—y simbólico — forma que adoptan las distintos tipos de capital al ser percibidos y reconocidos como legítimos-. Estas especies de capital no tienen el mismo peso ni cumplen el mismo papel y su orden de relevancia varía históricamente.

Para este autor, los agentes están distribuidos en el espacio social según el volumen global de capital que poseen, según la composición de su capital de acuerdo a los pesos relativos de cada especie, y según su trayectoria en el espacio social, que es la evolución en el tiempo dependiente del volumen y modo de adquisición del capital. La misma condición de clase, incluso la misma posición relacional en una estructura social en un momento dado, puede ser para distintos agentes el producto de distintos trayectos sociales y distintas estrategias, lo que impone una mirada diacrónica que atienda al origen e itinerario social. Los desplazamientos de los individuos en el espacio social no ocurren al azar sino en relación con un haz de trayectorias probables, trayectorias de clase colectivas -aunque eventualmente algunos individuos puedan salirse de la trayectoria modal de su clase de origen, a partir de apuestas y reconversiones entre los capitales poseídos-.

Bourdieu considera que la clase probable o "clase en el papel", definida por el sociólogo, se construye sobre la base de posiciones próximas en el espacio social proximidad que implica cierta probabilidad de que los agentes se asocien y movilicen como clase real-. Los agentes situados en posiciones cercanas en el espacio social comparten 
condiciones de existencia homogéneas y poseen un conjunto de capitales comunes, lo cual produce condicionamientos homogéneos, de los que tiende a resultar un habitus homogéneo.

La mirada sobre las clases sociales de Pierre Bourdieu procura poner en relación sus aspectos objetivos y subjetivos, es decir, las condiciones de existencia y las maneras de ver el mundo y de actuar de los agentes. El habitus es el concepto teórico clave que deviene en mecanismo explicativo crucial de los procesos sociales, que posibilita la comprensión y explicación del vínculo entre las prácticas y la estructura social. El habitus, sistema de disposiciones adquiridas por los sujetos a través de la experiencia duradera de una posición social, constituye tanto un principio cognitivo y evaluativo mediante el cual se perciben y aprecian las prácticas, como un principio generador y organizador de prácticas y representaciones. El habitus interioriza la posición social multidimensional, constituye formas básicas de visión del mundo y se exterioriza en prácticas.

Los agentes situados en posiciones objetivas próximas con toda probabilidad estarán constituidos subjetivamente por un habitus similar, que tenderá a generar prácticas semejantes, de las que resultará un estilo de vida determinado, unificado por el gusto. Este estilo de vida contribuye a configurar la misma clase, que es la contracara inescindible de las condiciones de existencia Pueden mencionarse entonces varias precauciones puestas de relieve por la perspectiva bourdieusiana que han de tenerse presentes para comprender las clases sociales: a) no reducirlas a la dimensión económica, sino tomar en cuenta la dimensión cultural y simbólica de las posiciones sociales, b) no limitar su estudio a una estructura estática, sino incorporar la mirada diacrónica de las trayectorias, c) ni tampoco analizar los factores por separado, sino examinar cómo se imbrican todas las propiedades determinantes.

La clase social no se define por una propiedad (aunque se trate de la más determinante como el volumen y la estructura del capital) ni por una suma de propiedades (propiedades de sexo, de edad, de origen social o étnico -proporción de blancos y negros, por ejemplo, de indígenas y emigrados, etc.-, de ingresos, de nivel de instrucción, etc.) ni mucho menos por una cadena de propiedades ordenadas a partir de una propiedad fundamental (la posición de las relaciones de producción) en una relación de causa a efecto, de condicionante a condicionado, sino por la estructura de las relaciones entre todas las propiedades pertinentes, que confiere su propio valor a cada una de ellas y a los efectos que ejerce sobre las prácticas (Bourdieu, 2012, p. 121).

Esta afirmación en La distinción [1979] de que la clase teórica se define no por una propiedad fundamental ni por una suma de propiedades sino por la estructura de relaciones entre todas las propiedades pertinentes, como pueden ser sexo, etnia o edad, implica una concepción estructural y relacional de la causalidad, en tanto red de elementos explicativos irreductible al efecto agregado de factores. A partir de esta consideración, es posible aproximarse a una vinculación de clase y género constitutiva de las desigualdades sociales. 


\section{Clase y género}

En La dominación masculina (2000 [1998]), Bourdieu considera al género como la forma paradigmática de la violencia simbólica, un principio de clasificación de primera importancia, distinguible de la clase y cuya naturalización ha sido más efectiva que la de ningún otro principio. En este trabajo de la última etapa del autor, el género deja de ser un factor más, como era en su obra clásica La distinción (2012 [1979]), para ser concebido como relativo a estructuras sexuales autonomizadas de las estructuras económicas a lo largo de la historia. El género y la clase interactúan de modo tal que cada principio modera los efectos que el otro ejerce en las prácticas. Las posiciones sociales como las ocupaciones están clasificadas y clasifican según género, al igual que otros principios de diferenciación tales como la etnia. Las complejas combinaciones de la dominación producidas por la intersección de distintos principios clasificatorios no pueden ser interpretadas automáticamente en términos de clase como en La distinción (Weininger, 2005), pero se mantiene el énfasis en el espacio social, en el que coexisten múltiples principios de diferenciación inseparables en su funcionamiento.

El género es uno de los principios de clasificación que opera en el espacio social. Las actividades, las cosas y los agentes son diferenciados a partir de la oposición entre lo masculino y lo femenino, división que resulta inseparable de un sistema de oposiciones homólogas como alto/bajo, delante/detrás, recto/curvo, duro/blando, fuera (público)/dentro (privado), etc. Estas oposiciones, diferentes pero afines entre sí, se apoyan mutuamente y dan lugar a transferencias prácticas, metáforas y connotaciones. Los esquemas de pensamiento registran como diferencias de naturaleza unas características distintivas que contribuyen a hacer existir. De este modo se "legitima una relación de dominación inscribiéndola en una naturaleza biológica que es en sí misma una construcción social naturalizada" (Bourdieu, 2000, p. 33).

El principio de diferenciación sexual y el principio de diferenciación social interactúan en la realidad, configurando el orden social y las disposiciones subjetivas. Los fenómenos relativos a la división sexual del trabajo de producción y reproducción constituyen aspectos privilegiados para la observación de las formas concretas de actuación conjunta de ambos principios. A partir del análisis etnográfico de los bereberes de la Cabilia, considerados como caso paradigmático de una tradición del área cultural mediterránea, Bourdieu (2000) observa que corresponde a los hombres, situados en lo exterior, lo público, lo oficial, lo alto y lo discontinuo, realizar las intervenciones breves, peligrosas y espectaculares que marcan rupturas en el curso normal de la vida, mientras que a las mujeres, situadas en lo interno, lo privado, lo bajo y lo continuo, se les adjudican los trabajos domésticos, generalmente invisibles, monótonos y humildes. Las estructuras de la división sexual actúan a través de 
principios prácticos que presionan propiciando que las funciones adecuadas para las mujeres sean una prolongación de las funciones domésticas, que el hombre tenga el monopolio de la manipulación de los objetos técnicos y de las máquinas y que una mujer no pueda tener autoridad sobre hombres.

El mercado matrimonial es el dispositivo central de las relaciones de producción y reproducción del capital simbólico, en el que las mujeres aparecen como símbolos u objetos que circulan de abajo hacia arriba, cuya función es la reproducción o incremento del capital simbólico y social poseído por los hombres, para su honor en la esfera pública. La violencia simbólica implica que no sólo las mujeres suelan ponerse de acuerdo con los hombres para aceptar los signos de una posición inferior, sino que en la imagen que se forjan de su relación con el hombre al que su identidad social está unida, las mujeres tengan en cuenta la imagen que el conjunto de los hombres y de las mujeres se harán aplicando los esquemas de percepción y de valoración socialmente compartidos. Esos esquemas comunes exigen que el hombre ocupe la posición dominante en la pareja, lo que explica que ellas suelan querer y desear a un hombre cuya dignidad esté claramente afirmada y demostrada en y mediante el hecho de que "las supera" visiblemente. Según Bourdieu en La dominación masculina, el modelo tradicional de matrimonio seguía siendo para las mujeres un medio privilegiado de adquirir una posición social.

Una parte muy importante del trabajo doméstico del que siguen ocupándose las mujeres tiene como fin mantener la solidaridad y la integridad de la familia conservando las relaciones de parentesco y el capital social. De la misma manera en que en las sociedades menos diferenciadas las mujeres eran tratadas como medios de intercambio, en la actualidad aportan una contribución decisiva a la producción y reproducción del capital simbólico de la familia, por ejemplo, al manifestar en su apariencia el capital simbólico del grupo doméstico. Al estar socialmente inclinadas a tratarse a sí mismas como objetos estéticos, se encargan con absoluta naturalidad, en la división del trabajo doméstico, de la estética y la gestión de la imagen pública y las apariencias sociales de los miembros de la familia, así como en el traslado de ese papel a las empresas, que les piden que desempeñen actividades de presentación y representación.

Las mujeres obreras están doblemente oprimidas por los efectos conjuntos de ambos ejes de desigualdad, pero esto no significa que las mujeres de otras clases estén menos oprimidas en tanto mujeres. La atención y el tiempo que las mujeres dedican a la propia presentación y cuidados del cuerpo es mayor a medida que se va elevando la posición social y entre aquellas insertas en ocupaciones en que las propiedades cosméticas puedan recibir algún valor, como ocurre en las profesiones de presentación y representación de la nueva pequeña burguesía (Bourdieu, 2012). Las que cuentan con un mayor capital corporal son las 
mujeres de las clases dominantes, que poseen las propiedades corporales más solicitadas y la capacidad de embellecerse mediante cuidados, todo lo cual confluye en una hexis corporal de soltura y seguridad del aspecto legítimo. Esto implica un efecto de naturalización y el refuerzo de los esquemas de percepción y apreciación de las mujeres en que se basa el poder simbólico de la dominación masculina.

En suma, para Bourdieu las mujeres de distintas posiciones de clase tienen en común su separación de los hombres por un coeficiente simbólico negativo que afecta negativamente lo que son y lo que hacen. Los hombres siguen dominando el espacio público y el campo de poder mientras que las mujeres permanecen en mayor medida en el espacio privado o en las extensiones de ese espacio, donde se reproduce la lógica de la economía de los bienes simbólicos. Seguramente algunos de los aspectos señalados por el autor deban ser repensados o cuestionados ante las transformaciones recientes en las relaciones de género, pero su aporte en torno a la dimensión simbólica de la desigualdad sigue siendo valioso para explicar lo que se perpetúa pese a —o mediante- los cambios. La interrelación entre clase y género debe estudiarse en cada situación y contexto histórico para establecer las singulares articulaciones de estos dos principios.

\section{Emancipación}

Para luchar contra la dominación es necesario comprender cómo opera la violencia simbólica. Esta se instituye a través de la adhesión que el dominado se siente obligado a conceder al dominador, debido a que los dominados aplican a las relaciones de dominación categorías construidas desde el punto de vista de los dominadores. Los esquemas de percepción y apreciación asimilados en la relación de dominación hacen que las clasificaciones resulten naturalizadas y la misma relación parezca natural. El concepto de ideología del marxismo, entendido como falsa conciencia, llevaría a esperar la liberación como efecto automático de la "toma de conciencia", ignorando la opacidad y la inercia que resultan de la inscripción de las estructuras sociales en los cuerpos. Para Bourdieu, en cambio, resulta ilusorio creer que la violencia simbólica puede vencerse sólo con las armas de la conciencia y la voluntad.

El hecho de que el poder simbólico no pueda ejercerse sin la contribución de los que lo soportan no significa que las mujeres sean responsables de su propia opresión por sus comportamientos de sumisión. Ha de explicarse la construcción social de las estructuras cognitivas que organizan los actos de construcción del mundo. Esa construcción práctica no es un acto intelectual consciente, libre y deliberado de un sujeto, sino que es el efecto de un poder inscrito de manera duradera en el cuerpo de los dominados bajo la forma de esquemas de percepción e inclinaciones. La revolución simbólica que reclama el movimiento feminista, 
del mismo modo que la lucha de clases no puede limitarse a una simple conversión de las conciencias y las voluntades ya que el fundamento de la violencia simbólica no reside en conciencias engañadas que bastaría con iluminar sino en inclinaciones modeladas por las estructuras de dominación que las producen.

\section{Discusión}

Wright y Bourdieu tienen perspectivas teóricas distintas, en cierto modo inconmensurables entre sí, pero pueden señalarse algunos tópicos en clave comparativa. Ambos autores concuerdan en que existen las clases y en que es de primordial importancia su estudio, pero mantienen importantes diferencias. Los mapas de clase de Wright serían para Bourdieu clases en el papel, clases cosificadas con límites arbitrarios que no se han vuelto reales mediante la asociación y movilización de los agentes. Inversamente, desde la mirada marxista, el concepto de clase de Bourdieu sería criticado por ser demasiado amplio -incluye desde las disposiciones corporales y los gustos de cualquier tipo, hasta la posesión de capital económico, pasando por las redes de relaciones sociales, el nivel cultural, el prestigio, etc. - y por carecer de criterios claros para demarcar fronteras de clase. La mirada marxista pondría el énfasis en la primacía de los procesos económicos en la definición de la clase, mientras que los aspectos culturales, como el gusto, los estilos de vida o las disposiciones corporales tenderían a tener el estatuto de efectos de la clase.

Aunque Wright complejice la clásica definición marxista de clase incorporando dimensiones como el control del capital, la autoridad en el proceso de trabajo o la posesión de calificaciones y credenciales educativas, mantiene lo fundamental de la tradición marxista en su definición de las clases, esto es, la posición en las relaciones de producción. Bourdieu, en cambio, contempla una noción de clase mucho más abarcadora. Considera que el terreno del consumo es por lo menos igual de importante que el de la producción para la formación de las clases. Los estilos de vida y los gustos son constitutivos de las clases y conforman su identidad cultural. En el mismo sentido, el capital no se circunscribe al económico, que pasa a ser una entre otras formas que puede asumir, junto con el capital cultural, el social, el simbólico y todos aquellos capitales específicos de los campos en que estén insertos los agentes.

También son diferentes las posturas de estos autores respecto a la relación entre clase y género. Para confrontarlas, es posible partir de los debates referidos a la posición de las mujeres en la estructura de clases y el problema empírico que se presenta en las investigaciones cuantitativas en estratificación y movilidad social respecto al tratamiento de las parejas heterogéneas — parejas donde ambos miembros son económicamente activos remunerados, pero tienen ocupaciones que remiten a distintas clases-. 
La controvertida perspectiva convencional de Goldthorpe afirmaba que la posición del jefe de familia con mayor responsabilidad en el mercado de trabajo determina la ubicación de clase de toda la familia. Debido a la menor participación de las mujeres en el trabajo asalariado, la ubicación de clase de ellas se identificaría con la de sus maridos. La exclusión de las mujeres en la medición de la clase social no presentaría dificultades cuando las mujeres no trabajan remuneradamente o cuando ambos miembros de una pareja son de una misma clase social. Sin embargo, cuando sus clases sociales difieren parece necesario admitir que la posición de clase de la mujer puede influir en las experiencias, identidad e intereses de clase del hogar. Baxter (1992) sostiene que, si bien no es adecuado centrarse solamente en la clase del individuo, un enfoque de clase de la familia debería tomar en cuenta la ubicación de clase de maridos y esposas, dado que las familias de clase heterogénea son cada vez más prevalecientes. De este modo, la unidad más apropiada para el análisis de clase dependería de la temática y características específicas de las investigaciones emprendidas. Gómez Rojas (2011), utilizando el esquema de clases de Goldthorpe, ha hallado que en los hogares con parejas heterogéneas en cuanto a su clase social, en que el esposo es de clase obrera pero la mujer no, la autoidentificación de clase obrera disminuye y aumenta la identificación con la clase media, lo cual cuestiona parcialmente la hipótesis convencional de Goldthorpe que sostiene que la clase social del esposo es lo más determinante en la autoidentificación de clase de las mujeres. Si para los varones tienen mayor relevancia las relaciones de clase directas, para las mujeres debido a sus menores ingresos, influirían tanto sus características propias como las derivadas del vínculo con otros miembros de la familia; estas se relacionan con la clase a través del consumo.

¿Qué podrían aportar Wright y Bourdieu sobre el problema de las parejas de clase heterogénea? A diferencia de Goldthorpe, Wright sí considera relevante para el análisis de clase tomar en consideración la heterogeneidad o diferencia entre las posiciones de clase de los miembros de la pareja. Desde la perspectiva de Wright, aunque los miembros de una familia compartan situaciones de consumo, las diferencias entre ellos respecto a sus posiciones de clase directas afectan la identidad de clase porque implican una serie de experiencias dentro del trabajo y pueden producir distintos intereses de clase. Entonces, la clase social de las mujeres no podría reducirse a la clase social de su esposo -su posición de clase mediada-, la cual sólo afecta parcialmente la identidad de clase de ellas mediante los intereses materiales. Asimismo, aunque el ingreso de las mujeres sea menor al de sus maridos, el carácter de clase de su trabajo remunerado puede influir en las estrategias familiares y en la clase de la familia como unidad. Wright argumenta que dos estructuras de clase, con las mismas pautas de relaciones directas, pero que difieren respecto de las relaciones mediatizadas, deberían considerarse como diferentes tipos de estructuras. 
Bourdieu no plantearía el problema de las parejas de clase heterogénea en esos términos. Como la clase social no descansa exclusivamente en la relación con el sistema productivo, sino que refiere a unas condiciones de existencia ampliadas que incluyen las distintas especies de capital y la trayectoria social, los miembros de una pareja con distintas ocupaciones y capitales económicos podrían o no pertenecer a una misma clase probable, dependiendo de sus habitus y estilos de vida. Para Bourdieu, a partir del panorama de todo el espacio social, deberían estudiarse las trayectorias familiares de clase, atendiendo a la estructura de capitales de sus miembros, su modo de adquisición y sus estrategias de reproducción. Desde su mirada, es posible interrogarse si el hecho de que mujeres y varones de una familia situados diferencialmente en el espacio social hace que difieran las disposiciones, experiencias y estilos de vida, o si por el contrario se trata de estilos de vida y habitus unificados, mientras que las diferencias refieren a una división sexual del trabajo propia de la familia, en relación con sus estrategias.

En cualquier caso, para que puedan observarse las desigualdades de género y clase, para Bourdieu es necesario un pensamiento relacional capaz de poner en relación la economía y los diferentes sectores del mercado de trabajo con la división sexual del trabajo. Podría así captarse la homología entre las estructuras de las posiciones masculinas y las femeninas en los diferentes espacios sociales. El mercado matrimonial, la economía de los bienes simbólicos, el trabajo doméstico y su función para la preservación e incremento de capital simbólico y social, son algunas de las dimensiones que podrían estudiarse para analizar la interrelación de clase y género. Entendiendo que los distintos principios de diferenciación operan conjuntamente en el espacio social, el análisis de las desigualdades debería intentar reconstruir la estructura de relaciones entre todas las propiedades pertinentes. Para Bourdieu, el vínculo entre clase y género más que de articulación sería de interpenetración.

En lo que respecta a las consecuencias metodológicas para la investigación, la teoría de Bourdieu resulta menos afín a las divisiones analíticas de aspectos indisolubles en la realidad, y por ende sus problemas de medición y técnicas de análisis no son los mismos que los de la sociología convencional de la movilidad social ni que los de Wright, quienes se valen primordialmente de abordajes cuantitativos multivariados basados en datos de encuesta. Bourdieu, si bien emplea las técnicas de análisis cuantitativas clásicas, recurre a un variado repertorio de técnicas y fuentes de información. En consonancia con sus supuestos teóricos y su concepción estructural de la causalidad, emplea la técnica estadística del análisis de correspondencias múltiples para observar la homología entre la estructura de posiciones de clase, los habitus y la estructura de estilos de vida. De este modo, produce una imagen de un espacio social atravesado por clase y género entre otros principios de diferenciación, cuya complejidad relacional se representa en el espacio factorial y tomatodos los factores 
simultáneamente, de forma tal que el peso de las demás relaciones "arrastra" consigo cada relación (cfr. Baranger, 2004).

Por último, para pensar la cuestión de la emancipación, los conceptos de violencia simbólica y habitus merecen ser tomados en cuenta para problematizar la mirada más ingenua de la toma de conciencia. En este sentido, cabría cuestionar los actores sociales racionales que supone Wright. Si no se cuenta con una noción como la de habitus, se soslayan las diferencias cualitativas en la constitución subjetiva y la racionalidad práctica de los agentes de distintas clases sociales. ¿Existe realmente algo como la conciencia de clase? ¿Abolir la dominación masculina puede depender de un cambio en la conciencia y voluntad de las mujeres? El concepto de violencia simbólica de Bourdieu resulta fundamental para comprender por qué los dominados no pueden simplemente "tomar conciencia" para luchar sin más por su emancipación. Las distintas conceptualizaciones sobre clase, género y emancipación ofrecen distintas explicaciones acerca de por qué las desigualdades son persistentes.

\section{Conclusiones}

El análisis de clase es una de las áreas más clásicas de investigación en sociología (cfr. Giddens, 1979) que sigue vigente en las investigaciones contemporáneas (Devine et. al., 2005). El conocimiento de la estructuración de las desigualdades de clase constituye un propósito en sí mismo como descripción de la organización básica de las relaciones sociales y de sus variaciones en el tiempo y en el espacio. Asimismo, permite explicar muchos otros fenómenos sociales que dependen de la clase, como prácticas, representaciones, sensibilidades, conflictos, acciones colectivas e incluso otras formas de desigualdad. Los estudios de estratificación y movilidad social prevalecientes a lo largo de gran parte del siglo pasado han considerado irrelevante la dimensión de género para sus propósitos. Sin embargo, en las últimas décadas han sido criticados precisamente por desconocer que dicha omisión afectaba la imagen de la estructura social que eran capaces de construir.

La relevancia del género en su imbricación con la estructura de clases ha sido planteada principalmente desde perspectivas feministas enfocadas en

(...) la crítica de la naturaleza fundamentalmente "generizada» de la sociedad moderna, que se refleja no sólo en la separación entre las esferas de actividad «pública» (masculina) y "privada» (femenina), sino también dentro de la esfera pública, como se demuestra, por ejemplo, en el grado de segregación por sexo en el mundo del empleo (Crompton, 1994, p. 189).

Sin embargo, el análisis de clase tendió a permanecer como un campo de estudios aparte, con sus propias problemáticas y discusiones teóricas, sin concederle la suficiente 
atención a que la estructura de clases está atravesada por el género y a que esto tiene consecuencias en los modos de investigar y representarse la estructura de clases.

Las perspectivas de Wright y Bourdieu ya son de algún modo clásicas en el análisis de clase, pero a la vez tienen un potencial para la renovación de la sociología convencional de la estratificación y movilidad social. Tanto las conceptualizaciones de clase de uno y otro autor como los modos de investigación que pueden llevarse a cabo desde sus enfoques difieren fuertemente. Los supuestos epistemológicos, las orientaciones valorativas y políticas respecto a las desigualdades y los fundamentos conceptuales de sus teorías conducen a problemas y estrategias de investigación específicos. Mientras que el enfoque de Wright resulta adecuado para investigaciones con objetivos y metodologías cuantitativas clásicas como el análisis multivariado, donde se definen criterios unívocos para asignar los individuos u hogares a una clase, el enfoque de Bourdieu requiere otras metodologías como el análisis de correspondencias o abordajes cualitativos para estudiar las relaciones entre posiciones en el espacio social. La complejidad se acrecienta cuando se pretende incorporar el género. Las propuestas de Wright acerca de la importancia de estudiar posiciones mediatas de clase 0 "clases sombra", y la concepción de Bourdieu sobre la multidimensionalidad de las clases sociales — que exige analizar la actuación conjunta de múltiples principios de diferenciación, proporcionan orientaciones para la renovación de los análisis de clase. El desafío consiste en que el género sea incorporado no como una simple variable que poner en relación con la clase sino como un aspecto constitutivo de la estructura social que se estudia.

\section{Referencias bibliográficas}

Álvarez Sousa, A. (1996). El constructivismo estructuralista: la teoría de las clases sociales de Pierre Bourdieu. Revista Española de Investigaciones Sociológicas 75, 145172.

Baranger, D. (2004). Epistemología y metodología en la obra de Pierre Bourdieu. Buenos Aires: Prometeo.

Baxter, J. (1992) Las mujeres y el análisis de clase: una perspectiva comparada. Política y Sociedad, núm. 11, 85-97.

Bourdieu, P. (2000). La dominación masculina. Barcelona: Anagrama.

Bourdieu, P. (2012 [1979]). La distinción. Criterios y bases sociales del gusto. Buenos Aires: Taurus.

Carabaña, J. (1995). Desigualdad y clases sociales: un seminario en torno a Erik O. Wright. Madrid: Visor-Argentaria. 
Castón Boyer, P. (1996). La sociología de Pierre Bourdieu. Revista Española de Investigaciones Sociológicas 76, 75-97.

Crompton, R. (1994). Estratificación. Una introducción a los debates actuales. Madrid: Editorial Tecnos.

Devine, F.; Savage, M.; Scott, J. y Crompton, R. (2005). Rethinking class: culture, identities and lifestyles. Basingstoke, Hampshire New York: Palgrave Macmillan.

Giddens, A. (1979). La estructura de clases en las sociedades avanzadas. Madrid: Alianza Editorial.

Goldthorpe, J. (1983). Women and class analysis: In defence of the conventional view. Sociology $17,465-488$.

Goldthorpe, J. (1992). Sobre la clase de servicio, su formación y su futuro. Revista Zona Abierta № 59-60, 229-263.

Gómez Rojas, G. (2010). Estratificación social, Hogares y Género: Incorporando a las Mujeres. Tesis de doctorado. Buenos Aires: FCS-UBA.

Gómez Rojas, G. (2011). Las mujeres y el análisis de clases en la Argentina: una aproximación a su abordaje. Lavboratorio. Revista de Estudios sobre Cambio Estructural y Desigualdad Social, Revista del Instituto de Investigaciones Gino Germani de la Facultad de Ciencias Sociales de la Universidad de Buenos Aires, año 12, núm. 24, 199-221.

Grusky, D. (2007). Social stratification: class, race and gender in sociological perspective. Avallon Publishing. Stanford: Avallon Publishing.

Longhi, A. (2005). La teorización de las clases sociales. Revista de Ciencias SocialesDepartamento de Sociología, Año XVIII. № 22, septiembre, 104-114.

Marx, K. y Engels, F. (1998). Manifiesto Comunista. Barcelona: Crítica.

Marx, K. (2003). El 18 Brumario de Luis Bonaparte. Buenos Aires: Prometeo.

Marx, K. (2006). El capital. Buenos Aires: Siglo XXI.

Rivas Rivas, R. (2008). Dos enfoques clásicos para el estudio de la estratificación social y de las clases sociales, en Espacio Abierto Cuaderno Venezolano de Sociología 17-3, 367-389.

Riveiro, M. y Castañeira, M. (2009). Comparando los esquemas de clases Wright y Goldthorpe en una encuesta nacional del 2007. Buenos Aires: 5as Jornadas de Jóvenes Investigadores. Instituto de Investigaciones Gino Germani, Facultad de Ciencias Sociales (UBA). 
Roemer, J. (1989). Teoría general de la explotación y de las clases. Madrid: Siglo XXI.

Salido Cortés, O. (2001). La movilidad ocupacional de las mujeres en España. Por una sociología de la movilidad femenina. Madrid: Centro de Investigaciones Sociológicas.

Sorensen, A. (1994). Women, family and class. Annual Reviews of Sociology 20, 27-47.

Wacquant, L. (2013). Symbolic power and group-making: On Pierre Bourdieu's reframing of class. Classical Sociology 13-2. Pp.: 274-291.

Weininger, E. (2005). Foundations of Pierre Bourdieu's class Analysis. En E., Wright (Comp.) Approaches to Class Analysis. (pp.: 82-118). Cambridge: Cambridge University Press.

Wright, E. O. (1983). Clases, crisis y estado. Madrid: Siglo XXI.

Wright, E. O. (1992). Reflexionando, una vez más, sobre el concepto de estructura de clases. En Revista Zona Abierta, núm. 59-60. Pp.: 17-126.

Wright, E. O. (1994[1985]). Clases. Madrid: Siglo XXI.

Wright, E. O. (1995). Análisis de clase. En J., Carabaña (Ed.) Desigualdad y clases sociales. Un seminario en torno a Eric O. Wright. (pp.:21-53). Madrid: Fundación Argentaria.

Wrigth, E. O. (2002). The Shadow of Exploitation in Weber's Class Analysis. American Sociological Review, 67, 832-853.

Wright, E. O. (2005). Conclusion: if "class" is the answer, what is the question? En E. O., Wright (Comp.). Approaches to Class Analysis. (pp.: 180-192). Cambridge: Cambridge University Press.

Wright, E. O. (2010 [1994]). Preguntas a la desigualdad. Ensayos sobre análisis de clase, socialismo y marxismo. Bogotá: Editorial Universidad del Rosario. 